\title{
PAUTAS GENERALES PARA LA CREACION DE UNA NAVE DE ALMACENAMIENTO MODULAR DESTINADA A PRODUCTOS NO PERECEDEROS.
}

\author{
GENERAL GUIDELINES FOR THE CREATION OF A MODULAR STORAGE BUILDING PRODUCTS NOT \\ INTENDED FOR PERISHABLE. \\ Carlos Henry, Berbeo García. ${ }^{1}$ \\ Oscar, Palacio León. ${ }^{2}$ \\ ${ }^{1}$ Ingeniero Industrial, Estudiante de posgrado, Gerencia Integral en Logística, Facultad de \\ Ingeniería Universidad Militar Nueva Granada, Bogotá D.C, Colombia. \\ Henryber0@gmail.com \\ ${ }^{2}$ Ing. Industrial, M.Sc., Profesor asistente, Facultad de Ingeniería, Investigador grupo ITE, \\ Universidad Militar Nueva Granada, Bogotá D.C, Colombia. \\ Oscar.palacio@unimilitar.edu.co
}

\section{Resumen:}

El deterioro del medio ambiente y particularmente los cambios en el clima, obliga al conjunto de la sociedad y a todos los sectores productivos y económicos que lo provocan a una reorientación profunda de las pautas de producción y consumo.

El presente documento es una guía metodológica para el diseño de una nave industrial, el cual se basará en conceptos actuales de construcción sostenible, diseños amigables con la naturaleza dirigidos cada vez a una producción que aproveche de manera mesurada y adecuada todos y cada uno de los recursos que el medio ambiente brinda.

Palabras clave: Nave de almacenamiento, construcción sostenible, construcción ligera, constructibilidad, ultimo planificador.

\begin{abstract}
:
The deterioration of the environment, particularly changes in climate, forces the entire society and all productive and economic sectors that give rise to a major redirection of production and consumption patterns.

This paper is a methodological guide from the design of an industrial building is based on current concepts of sustainable construction, nature friendly designs aimed anticreasing production to take advantage of a measured and appropriate each and every one of the resources that the environment provides.
\end{abstract}

Key words: storage shed, green construction, lean construction, construction, Last Planner 


\section{INTRODUCCIÓN.}

En la actualidad y con la evolución continua de los mercados, el desarrollo de los nuevos sistemas de producción y el constante crecimiento mundial, es necesario contemplar métodos o mecanismos de afrontar los nuevos retos que trae todo este proceso de comercialización cambiante[1], es por ello que uno de los puntos más importantes a definir es el diseño de nuevas plantas de manufactura, las cuales serán el pilar sobre el cual se regirán los procesos rápidos en cuanto a capacidad de producción, livianos en cuanto a respuestas a cambios o modificaciones y robustos frente a la competencia capaces de producir a un ritmo o exigencia acelerada de producción [2]; el presente documento busca introducir al profesional o al lector a desarrollar pasos secuenciales para diseñar plantas que cuenten con estas características:

Las creación de plantas de producción industriales son componentes fundamentales de las redes de niveles múltiples necesarias para la excelencia en la cadena de uso [3]. Por lo tanto, cada organización en la cadena de la demanda debe planificar sus instalaciones tomando en consideración sus asociados en la red de valor [4], una adecuada planeación de instalaciones industriales a lo largo de la cadena de valor asegura que el producto se manufacturara y entregara a entera satisfacción del cliente final, por lo tanto y según lo descrito por el autor Tompkins, en cuanto a que todas las naves industriales inmersas en las redes de valor deberán contemplar las siguientes características: Flexibilidad, modularidad, facilidad de actuación, adaptabilidad y operatividad selectiva, todo lo anterior marcado en un enfoque dirigido a al adecuado uso del medio ambiente y a la conservación de recursos naturales[5].

En la actualidad las plantas industriales son uno de los sectores más desarrollados de un país, además de ser el mayor productor de empleos para la sociedad actual. Los grandes maestros de la ingeniería han toma do el diseño de una planta como el factor primordial para un eficiente desarrollo industrial [6].

Toda planta industrial es la fusión perfecta entre el Hombre y la Máquina, trabajando así como uno, donde la función principal del hombre es la obtención del mayor rendimiento de las Máquinas [7].

Hoy debemos situarnos en el espacio ambiental, no podemos dejar de lado el triste envejecimiento de nuestro planeta, causado por nuestros derroches de tecnología y uso irracional de los recursos naturales ; utilizamos el agua contaminándola con todos los procesos industriales, talamos arboles indiscriminadamente sin hacer un control de resiembra y el uso inconsciente de combustibles, hace que cada vez el ser humano encamine sus esfuerzos hacia el aprovechamiento de las diferentes fuentes que la naturaleza nos provee, por ello debemos introducir nuevos conceptos de construcción limpia que nos ayuden a mitigar el impacto que dicha evolución causa al medio ambiente [8]. 


\section{NORMATIVIDAD.}

Para dar inicio al desarrollo del diseño de las naves industriales debemos partir de la normatividad y aplicación de las leyes que regirán la evolución del proyecto, Las empresas deben tener estipuladas políticas ambientales orientadas a la prevención y minimización de impactos y riesgos ambientales a los seres humanos y al ambiente garantizando la protección ambiental de la localidad en que se encuentra ubicada mediante la optimización del uso de los recursos naturales, materias primas y energía, prevenir y minimizar la contaminación, los residuos y los impactos ambientales, adoptar tecnologías limpias y prácticas de mejoramiento continuo, apoyándose en estructuras como producción más limpia y promoción de la autogestión y autorregulación ambiental empresarial [9].

\section{LEGISLACION COLOMBIANA.}

La constitución del 91, llamada la constitución verde, estableció responsabilidad de parte del estado y de las empresas el cuidado del medio ambiente y de sostener un ambiente adecuado para la comunidad que las rodea. Algunos de estos artículos son:

- Artículo 8. Es obligación del Estado y de las personas proteger las riquezas culturales y naturales de la Nación [10].

- Artículo 79. Todas las personas tienen derecho a gozar de un ambiente sano. La ley garantizará la participación de la comunidad en las decisiones que puedan afectarlo. Es deber del Estado proteger la diversidad e integridad del ambiente, conservar las áreas de especial importancia ecológica y fomentar la educación para el logro de estos fines [11].

- Artículo 80. El Estado planificará el manejo y aprovechamiento de los recursos naturales, para garantizar su desarrollo sostenible, su conservación, restauración o sustitución. Además, deberá prevenir y controlar los factores de deterioro ambiental, imponer las sanciones legales y exigir la reparación de los daños causados [12].

Basándonos en la ordenanza territorial a distrital referente a los aspectos de normativos de construcción enfocados a los desechos o escombros [13], para tal fin tenemos la siguiente legislación ambiental para la remoción de escombros [14], DECRETO 357 DE 1997 [15].

Artículo $\mathbf{1}^{\mathbf{}}$... Para fines de este Decreto se tendrán las siguientes definiciones:

Escombros: Todo residuo sólido sobrante de la actividad de la construcción, de la realización de obras civiles o de otras actividades conexas complementarias o análogas.

Materiales de construcción: Arenas, gravas, piedra, recebo, asfalto, concretos y agregados sueltos, de construcción o demolición. Capa orgánica, suelo y subsuelo de excavación. Ladrillo, cemento, acero, hierro, mallas, madera, formaleta y similares.

Estaciones de transferencia: Son los lugares en donde se hace el traslado de escombros de un vehículo recolector a otro, con mayor capacidad, que los transporta hasta su disposición final en las escombreras. 
Escombrera distrital: Es el lugar determinado por el Distrito para la disposición final de los materiales de que trata el presente Decreto.

Operador de escombreras o de estaciones de transferencia: Es la persona, comunidad, unión temporal o consorcio que celebra un contrato con el Distrito Capital, con el objeto de administrar escombreras o estaciones de transferencia.

\subsection{LICENCIA DE CONSTRUCCIÓN.}

Una licencia urbanística, es una autorización previa para urbanizar, parcelar, subdividir o edificar un predio. Para inmuebles existentes se requiere licencia para reforzarlo o modificarlo, ampliarlo o adecuarlo a otro uso, igualmente para el cerramiento de cualquier inmueble. Dentro de las cuales se conocen:

* De urbanización.

* De parcelación.

* De subdivisión y sus modalidades.

* De construcción y sus modalidades.

* De intervención y ocupación del espacio público (su estudio y trámite corresponde a la oficina de planeación municipal o distrital).

En caso que una construcción no posea la licencia, se debe solicitar el reconocimiento de la construcción el cual hará las veces de licencia, sin perjuicio de las sanciones respectivas por dicha infracción urbanística. No se requiere cuando se realicen obras de mantenimiento o de reparaciones locativas de cualquier edificación, destinadas a mantener el inmueble en las debidas condiciones de higiene y ornato [16].

Para las solicitudes de licencia clasificadas bajo las categorías III Medía Alta Complejidad y IV Alta Complejidad de que trata el artículo 18 del presente decreto, copia de la memoria de los cálculos y planos estructurales, de las memorias de diseño de los elementos no estructurales y de estudios geotécnicos y de suelos que sirvan para determinar el cumplimiento en estos aspectos del Reglamento Colombiano de Construcción Sismo resistente -NSR-10, y la norma que lo adicione, modifique o sustituya, firmados y rotulados por los profesionales facultados para este fin, quienes se harán responsables legalmente de los diseños y estudios, así como de la información contenida en ellos. Para las solicitudes de licencia clasificadas bajo las categorías I Baja Complejidad y II Media Complejidad de que trata el artículo 18 del presente decreto únicamente se acompañará copia de los planos estructurales del proyecto firmados y rotulados por el profesional que los elaboró [17]. 


\subsection{MODALIDADES DE LICENCIA.}

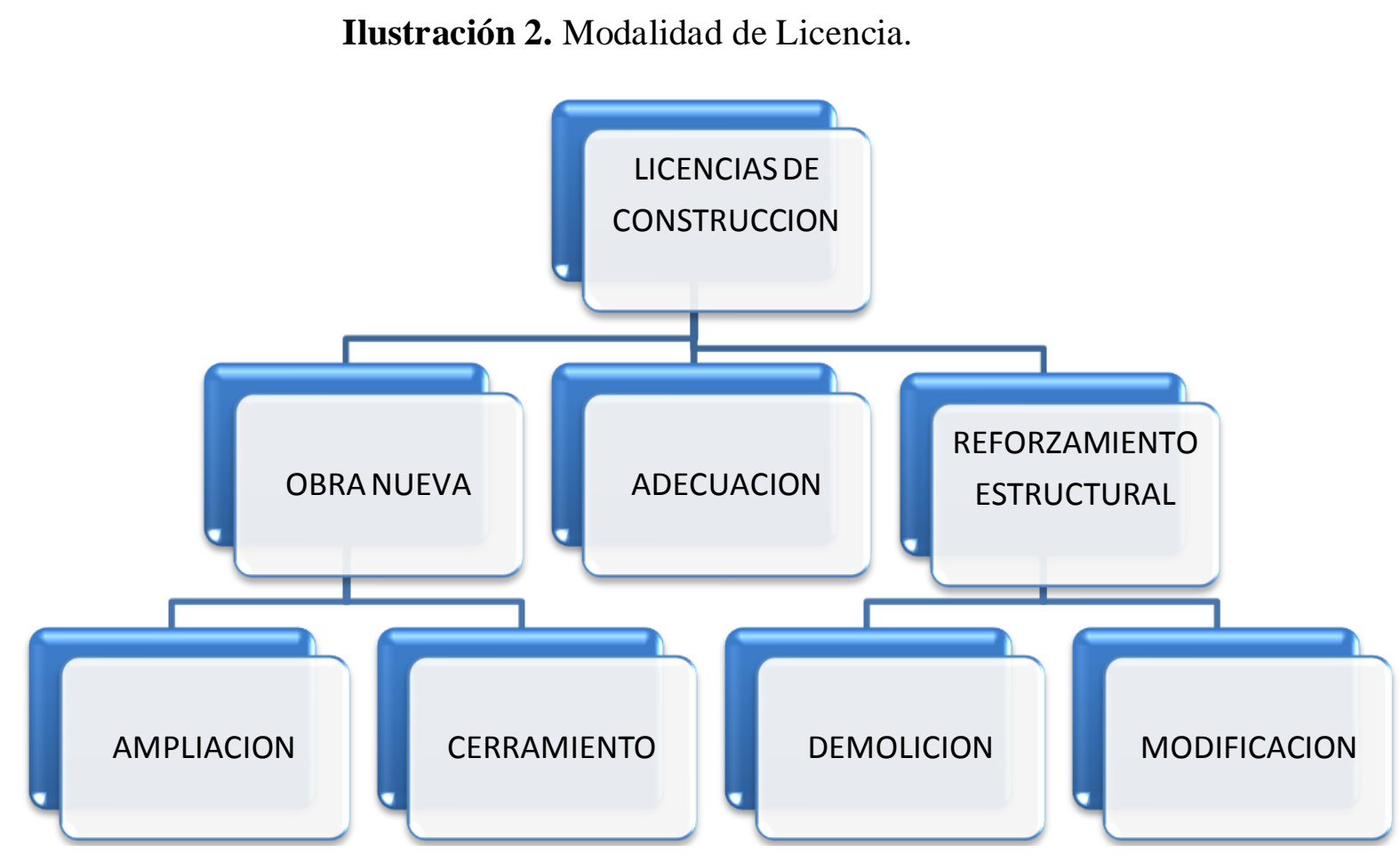

Fuente: http://www.curaduria3bogota.com

3.2.1. Obra Nueva: es la autorización para desarrollar obras en un terreno no construido [18].

3.2.2. Ampliación: es la autorización para aumentar el área construida en edificaciones ya existentes que tengan licencia de construcción [19].

3.2.3 Adecuación: es la autorización para cambiar el uso de una edificación o parte de ella, sin alterar la construcción original [20].

3.2.4. Modificación: es la autorización para cambiar el diseño arquitectónico o estructural de una edificación ya existente sin aumentar el área construida [21].

3.2.5. Restauración: es la autorización para desarrollar obras con el fin de recuperar y adecuar una edificación declarada como bien de interés cultural o parte de ella, manteniendo su uso original o permitiendo el desarrollo de otro garantizando su conservación [22].

3.2.6. Demolición: es la autorización para derribar total o parcialmente una o varias edificaciones existentes en uno o varios predios. Esta modalidad de licencia deberá tramitarse de manera simultánea con otra modalidad [23].

3.2.7. Cerramiento: es la autorización para encerrar de manera permanente un predio de propiedad privada [24]. 


\subsection{Normativa sismo resistente.}

Colombia cuenta con un completo contenido en materia de requisitos mínimos para garantizar el fin primordial de la norma en su conjunto previsto en la Ley 400 del 97: amparar las vidas humanas ante un sismo fuerte y proteger el patrimonio del Estado y de los ciudadanos...”, “...De esta forma, las estructuras construidas bajo la NSR-10 deben ser capaces de resistir temblores de poca intensidad sin daño, temblores de mediana intensidad sin daño estructural y un temblor fuerte sin colapso..." [25], algunos de las pautas a tener en cuenta son:

- Nuevo Mapa de Sismicidad elaborado por la Red Sismológica Nacional adscrita al Ingeominas, para esto se registraron 22.000 eventos sísmicos (NSR-98 tenía 13mil) adicionales desde el año 1995 hasta el 2009 logrando una estimación más precisa. De los cuales 100 tuvieron una magnitud de Richter mayor de 5.0.

- Presenta ajustes en las excavaciones y estabilidad de los taludes con consideraciones en sus estructuras, en las evaluaciones geotécnicas de los efectos sísmicos, en los sistemas constructivos de las cimentaciones, sus excavaciones y los muros de contención.

- También para la rehabilitación sísmica de edificios se incluyeron amenazas de origen sismo geotécnico y reforzamiento de edificaciones.

\section{INICIATIVAS DE CAMBIO.}

En la historia reciente hay muchas iniciativas con respecto al tema ambiental. Los protocolos de Kioto y Montreal en 1997, le dieron relevancia al tema y dieron un gran paso frente a las sanciones que debíamos tomar para detener el deterioro de la atmosfera. En diciembre se realizo la cumbre ambiental en mundial en Copenhague. La preocupación principal sobre el cambio climático y la incidencia de los gases producidos por nuestra civilización [26].

Teniendo en cuenta la normatividad que debemos seguir para dar inicio al desarrollo de los pasos escalonados del diseño de naves de almacenamiento generales para productos no perecederos, que mejor manera de comenzar que emitir una definición particular de lo que a naves de almacenamiento o almacenes se refiere para así, desarrollar la secuencia y entendamos todo lo que a ella se refiere. 


\section{NAVE INDUSTRIAL.}

\subsection{Definición.}

Una nave industrial es una construcción propia para resolver problemas de alojamiento y operación de la industria [27], se puede definir también como una instalación física o edificación diseñada y construida para realizar actividades industriales de de producción transformación, manufactura, ensamble. Procesos industriales, almacenaje y distribución [28].

Fig 1. Esquema de nave industrial.

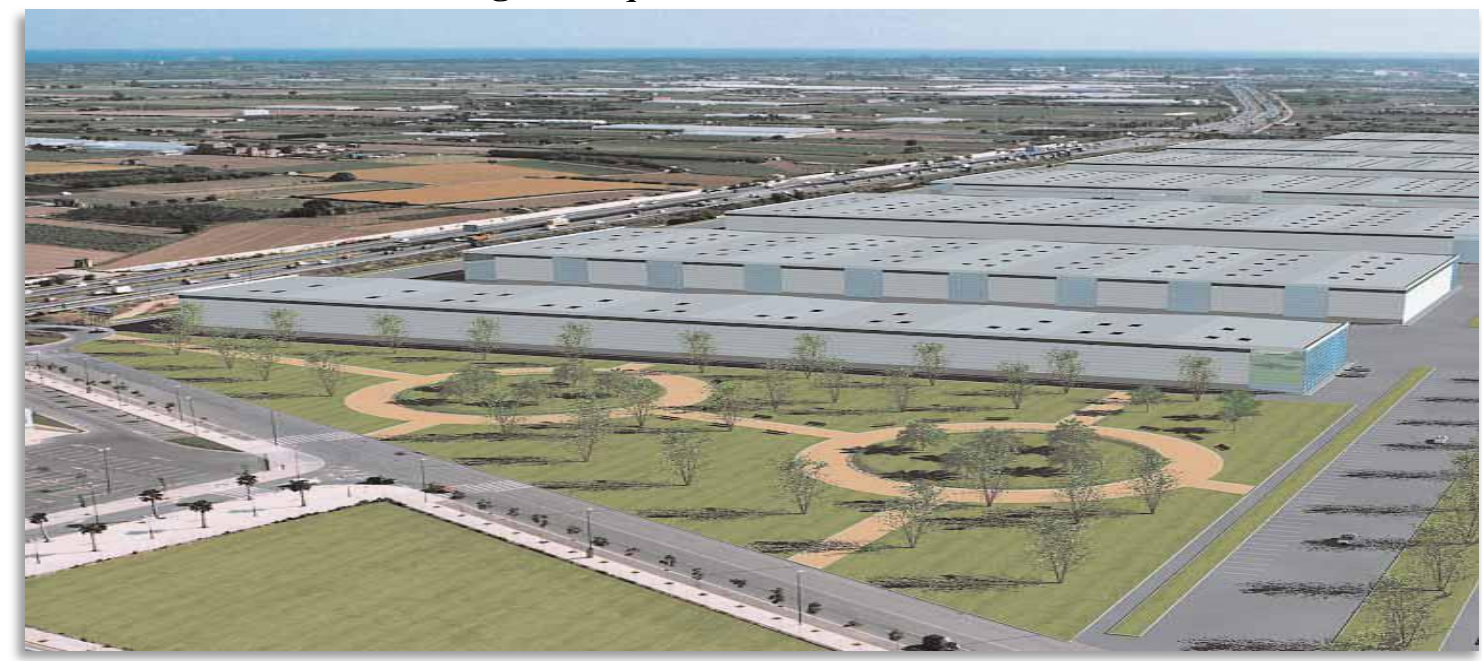

Fuente: http://www.mecalux.es/Diseño/Naves Industriales.

Encaminados al concepto ambiental como directriz de este documento nos enfocaremos en desarrollar la metodología a seguir para la creación de naves de almacenamiento tipo leed construcción, que sean amigables con la naturaleza y su preservación.

\subsection{Fundamentos leed construcción.}

Leed Construcción es una nueva forma de gestionar la construcción [29], el objetivo, principios y técnicas de construcción sin pérdidas en su conjunto constituyen la base para un proceso de ejecución de proyectos nuevos.

Figura 2. Sello de certificación que tan "verde es su proyecto"

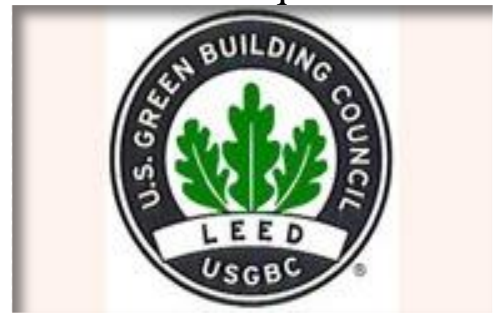

Fuente: http://www.google.com.co/imgres

Es un sistema para certificar el diseño de la construcción y la operación de edificios ambientalmente sostenibles. Está basado en un sistema de puntuación que abarca diferentes criterios como la eficiencia energética, el aprovechamiento del agua, los materiales utilizados, el 
impacto en el entorno y la calidad ambiental interior. Se trata de un sistema para certificar el diseño, construcción y operación de edificios ecológicamente sostenibles [30].

A diferencia de los enfoques actuales de la gestión de construcción (incluidos los de diseño y construcción), Leed Construcción provee la base para las operaciones basados en los proyectos que entrega el sistema [31].

\subsection{Propósitos Del Leed.}

Aunque el concepto de leed abarca múltiples aplicaciones de la vida cotidiana, a continuación mencionaremos las más relevantes con respecto a nuestro tema a tratar sobre naves de almacenamiento [32]:

a) Evitar el greewashin o pretensiones falsas o exageradas.

b) Liderar un proceso de diseño integral en el proyecto.

c) Contribuir con la reducción del impacto negativo al medio ambiente.

d) Promover procesos inteligentes, eficientes, rentables y saludables.

e) Mejorar las condiciones ambientales de los ocupantes.

f) Mejorar la operación y costo en el mantenimiento de los edificios.

Figura 3. Lo que mide el Leed.

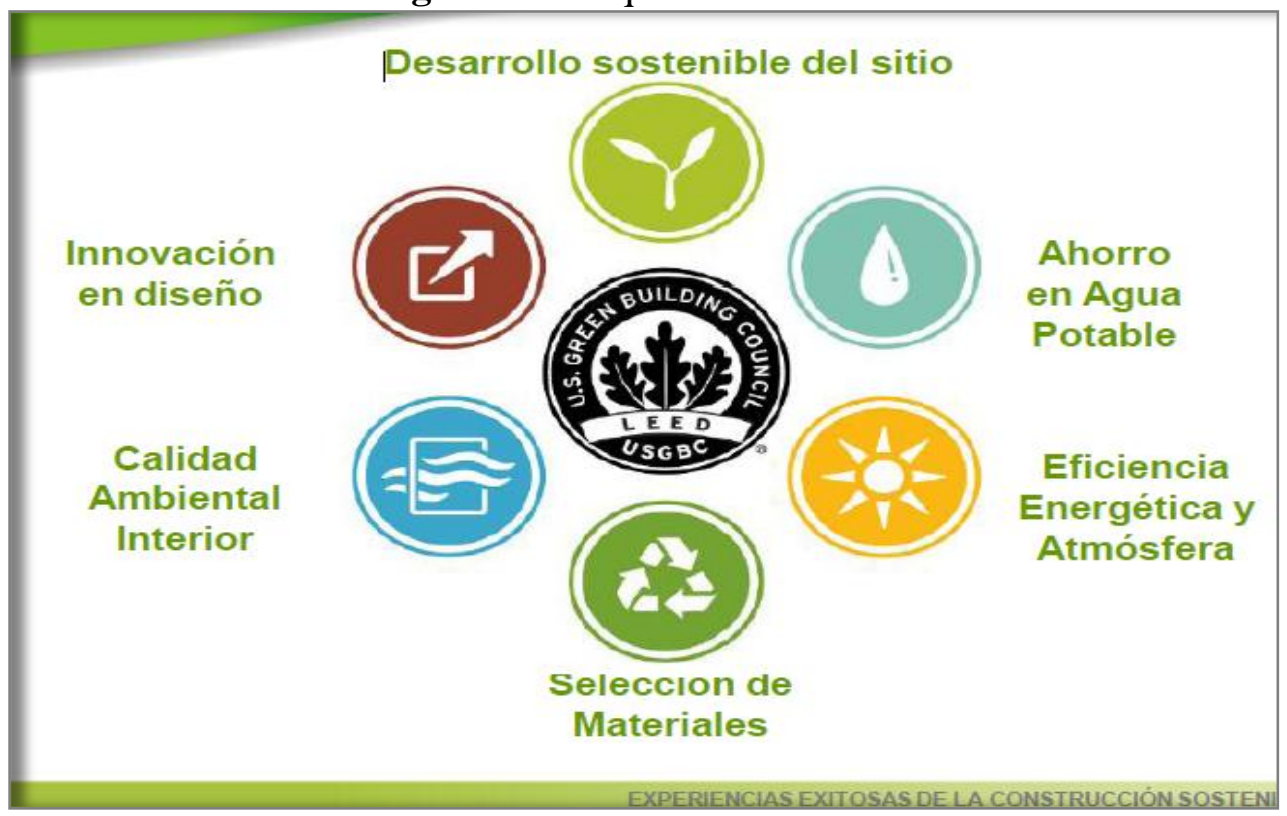

Fuente: Construcción sostenible - expocamacol.

El sistema del Último Planificador es una herramienta para controlar interdependencias entre los procesos y reducir la variabilidad entre éstos y por ende, asegurar el mayor cumplimiento posible de las actividades de la planificación dentro de la filosofía "Leed Construcción".[33], es decir que cada eslabón en el proceso debe estar unido a otro y todos deben conocer la debida secuencia. 


\section{CONCEPTOS FUNDAMENTALES PARA EL DISEÑO DE ALAMACEN [34].}

- Cada producto posee un equipo de almacenamiento adecuado en función de su tamaño, intensidad de flujo y cantidad de stock.

* Existe un equipo de manipulación adecuado para manipular los productos en función de la forma de manipulación, a la altura a la que se opera, peso y velocidad de flujo.

* La cantidad y ubicación de los andenes/muelles depende de la naturaleza del lugar del almacenamiento.

* El número y tipo de los equipos de manipulación dependerá de la concepción del sistema de operación interno del almacén.

* La distribución de los productos al interior del almacén condiciona fuertemente la producción del mismo.

* El volumen interior del almacén es de aproximadamente, entre 3 y 6 veces el volumen de stock requerido para almacenar, dependiendo del flujo.

\subsection{CARACTERISTICAS FISICAS DEL ALMACEN.}

\subsubsection{LOCALIZACIÓN.}

La Localización es un estudio de soluciones múltiples, es decir, que existen más de una localización factible adecuada que puede hacer rentable, el proyecto debe considerarse la evolución de los factores en el tiempo ya que una solución óptima en las condiciones vigentes puede no serlo en el futuro. Debe por lo tanto estudiarse la localización que optimice los flujos de ingresos y egresos en la vida del proyecto y esa sería la localización ideal [35].

Con base en la filosofía leed la ubicación de la planta o edificación es fundamental en la práctica de la sostenibilidad.

El daño al medio ambiente se puede prevenir escogiendo sitios previamente desarrollados con acceso a trasporte público y acceso a servicios básicos sin utilizar vehículos, se deben estudiar impactos en la hidrología del lugar, los ecosistemas y la vegetación existente [36].

Para prevenir el efecto invernadero, escoger materiales de construcción mas amigables con el medio ambiente. 
Figura 4. Ubicación del proyecto, importante para mitigar el impacto ambiental.

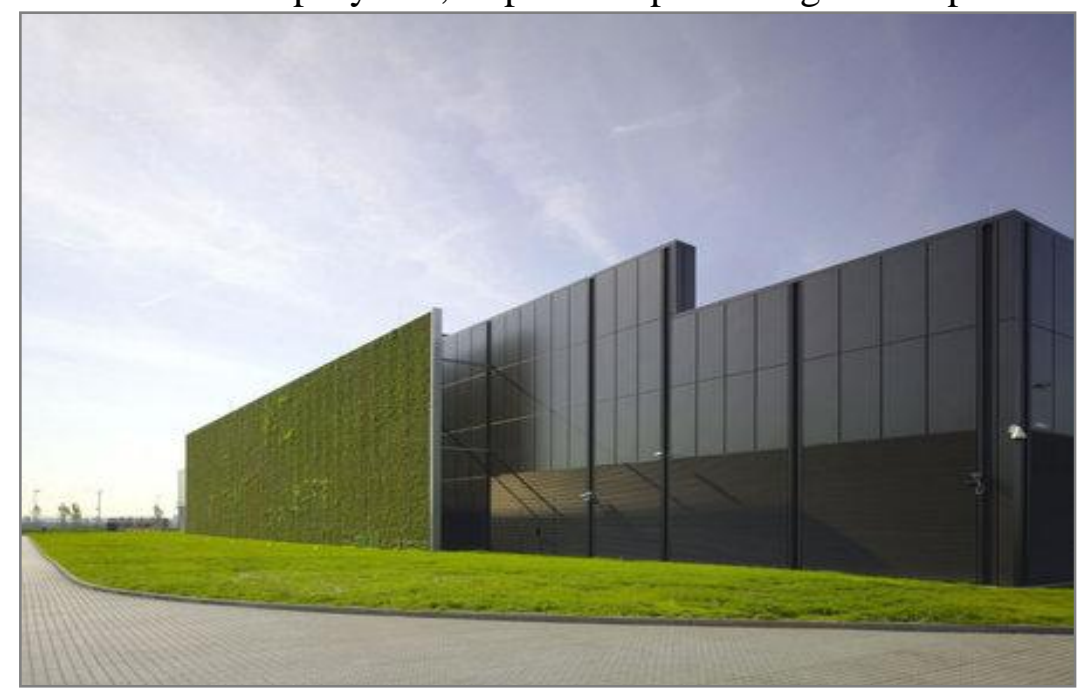

Fuente: http://www.google.com.co/search

Pensando verde, algunos de los factores de mayor relevancia al momento de escoger el lugar para construir una nave de almacenamiento son; reducir la contaminación generada por la construcción, proteger ecosistemas, minimizar el uso del automóvil [37].

\subsection{ETAPAS A TENER EN CUENTA PARA EL DESARROLLO DE LA OBRA.}

En el presente apartado se fija un proceso de ejecución de las obras, con objeto de definir y ordenar el conjunto de las operaciones a realizar en dicha construcción. Detallando y especificando la manera de ejecutar cada una de ellas, manteniendo el orden y coordinación entre las diferentes operaciones [38].

6.2.1. Movimiento de tierras: La ejecución comenzará con una limpieza general del terreno, quitando todo tipo de tierra vegetal y estorbos. Todo el material recogido será transportado por camiones hacia el vertedero.

Figura 5. Proceso de limpieza del área.
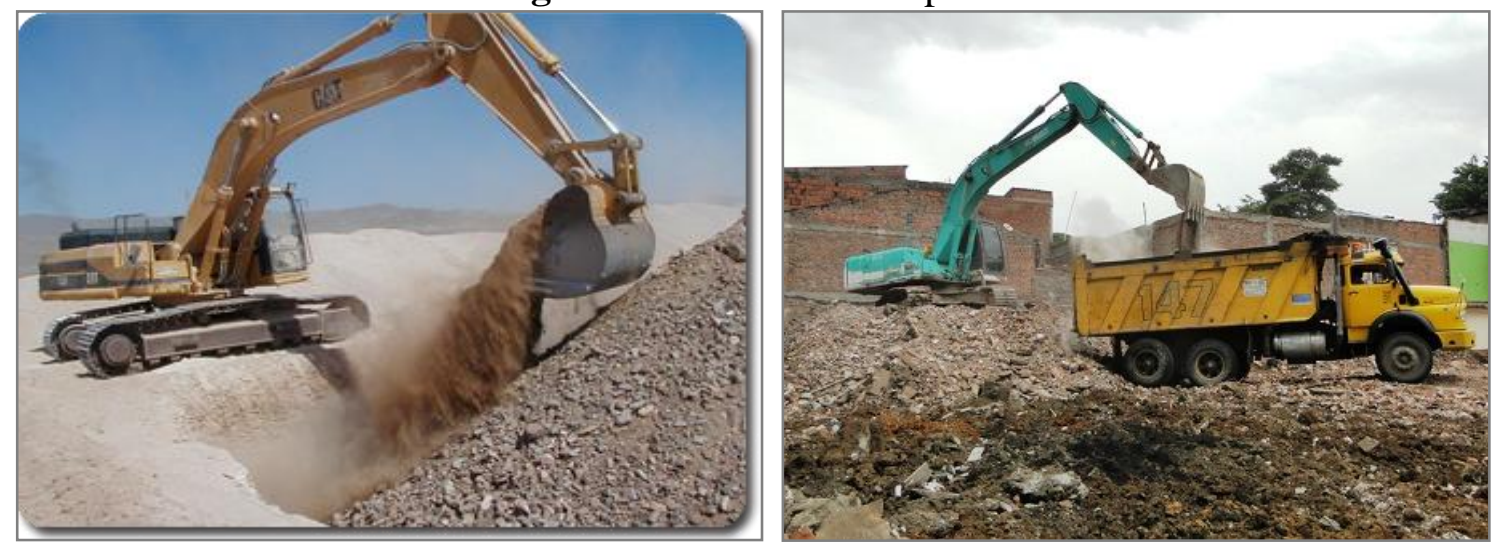

Fuente: http://www.google.com/search 
Se colocaran unas vallas de 2 metros de altura alrededor del perímetro de la parcela para realizar las tareas de la obra sin causar ningún problema y evitar que personas ajenas a la misma tengan accidentes [39].

\subsubsection{Cimentación:}

La cimentación tendrá lugar después del movimiento de tierras. Se harán dos tipos de cimentación:

1.- Pozos para las zapatas.

2.- Riostras.

Figura 6. Proceso de cimentación.

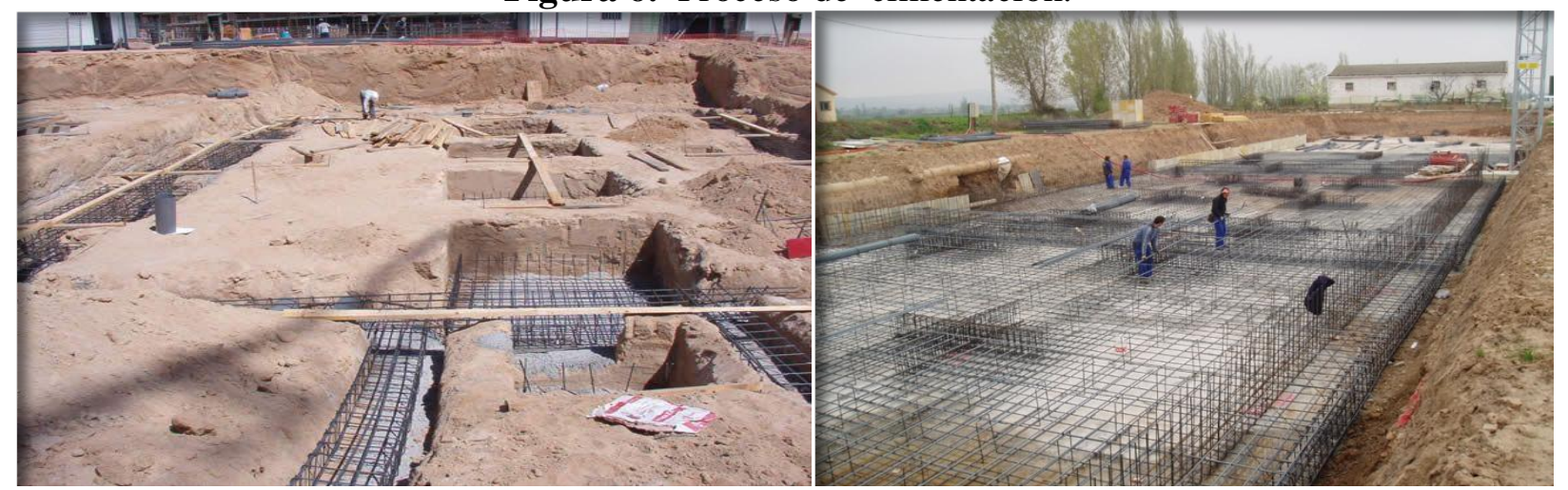

Fuente: http://www.google.com/search

\subsubsection{Estructura:}

Una vez tengamos toda la cimentación realizada, se procederá a la ejecución de la estructura, que básicamente está formada por unas correas que nos servirán para realizar la construcción del tejado, apoyadas en la estructura de las cerchas, que a la vez están sujetas por los pilares de la nave, y para la sujeción de la fachada, unas vigas apoyadas en los pilares.

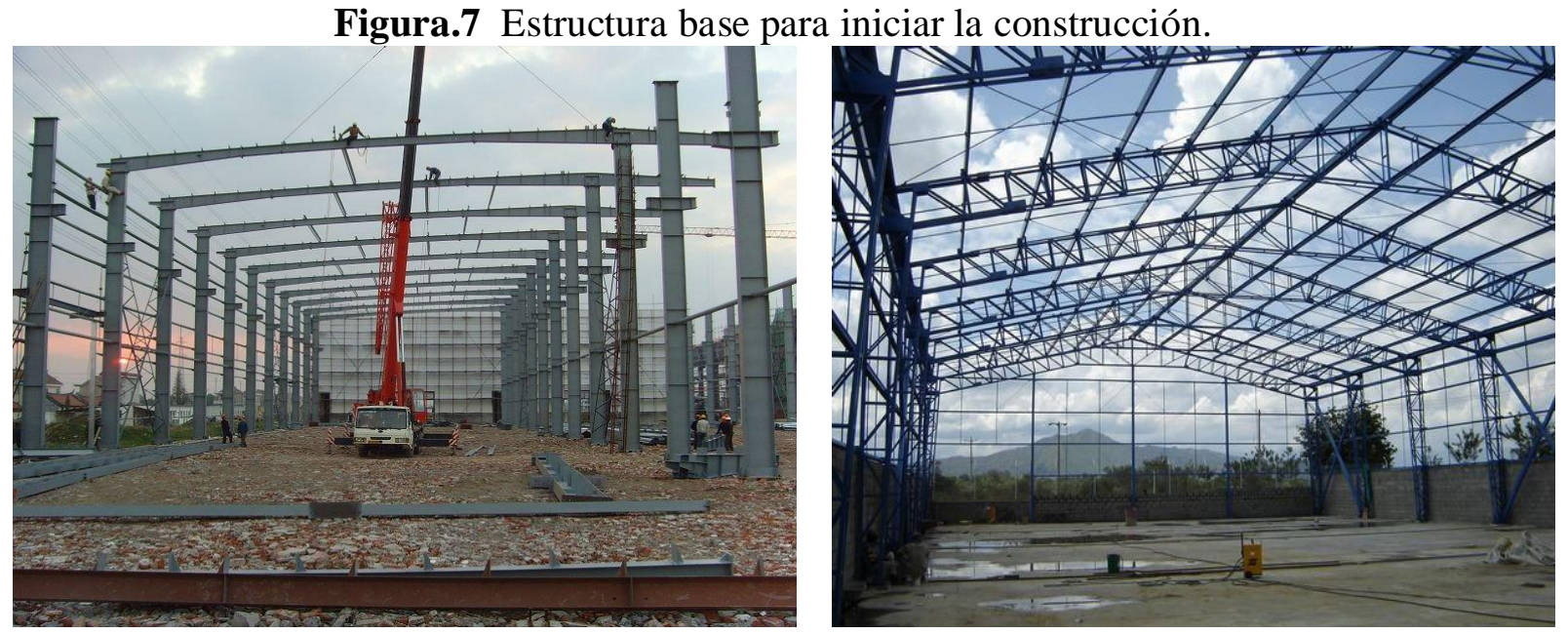

Fuente: http://www.google.com/search 


\subsubsection{Elementos Que Componen La Estructura.}

\subsection{Vigas de techo:}

Según se requiera, los arreglos de la nave pueden darse con la viga der techo en posición normal o invertida, y esta a su vez pueden ser sencillas o dobles.

El ancho medio de la línea de centro entre columnas, puede oscilar desde 18 a 30m, por lo tanto, puede alcanzarse anchos desde $36 \mathrm{~m}$ hasta los $60 \mathrm{~m}$. para naves industriales con doble viga de techo.

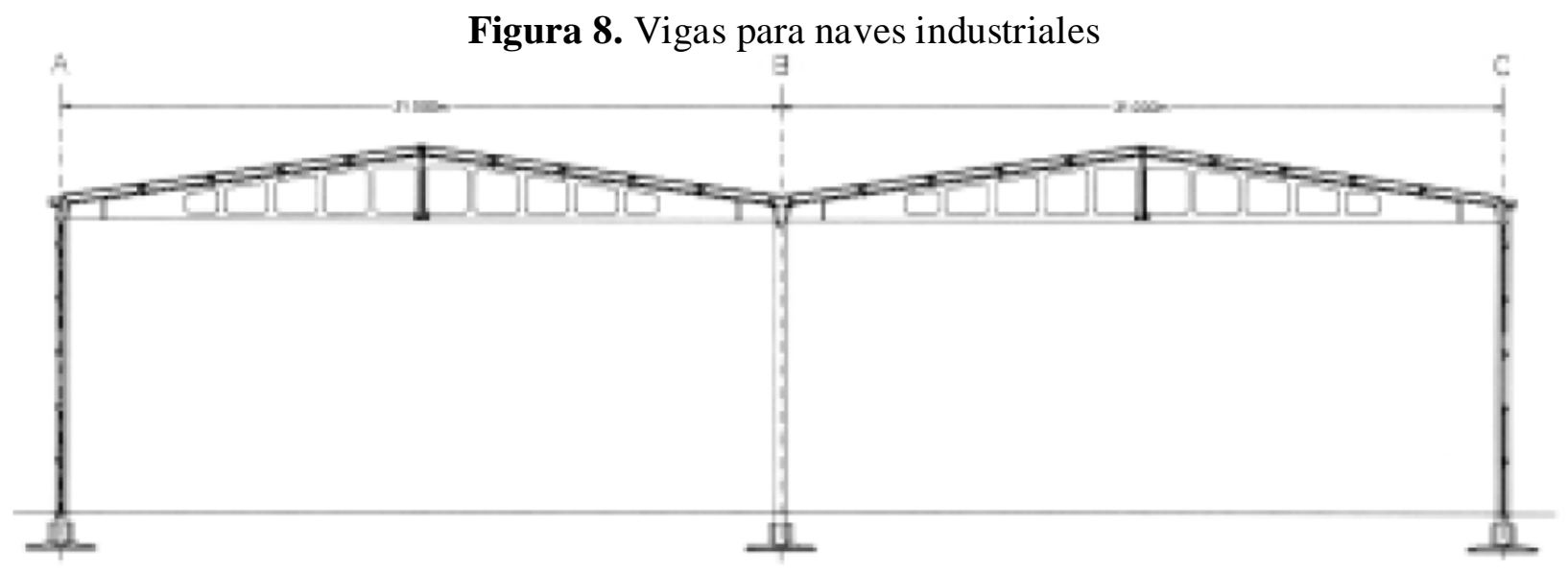

\section{Sistema estándar para naves industriales (hasta $31 \mathrm{~m}$ de luz)}

Fuente: Catalogo Naves Industriales - Empresa Holcim (costa rica)

\subsection{Paredes:}

El sistema de naves industriales permite la solución de paredes con dos sistemas diferentes:

Horizontales

* Verticales

Figura 9. Tipos de paredes.

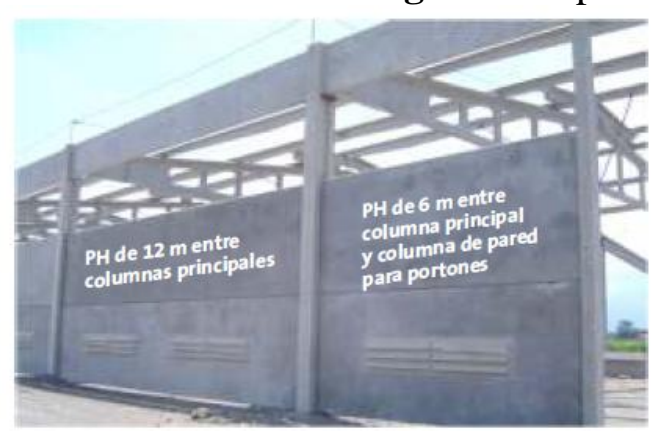

Fachadas con Paneles Horizontales: PH@12 m dentro de las Ilaves de columnas principalesy PH@6 m entre Ilave de columna principaly llave de columna de pared.

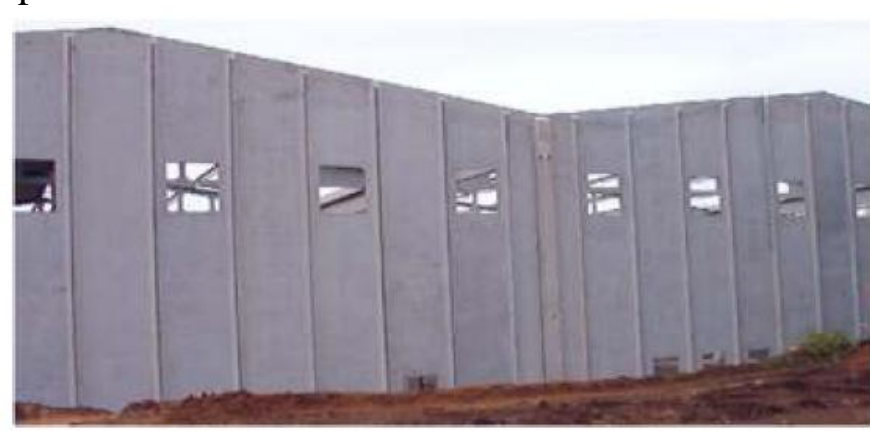

Fachadas con Paneles Verticales: PV se colocaron con la nervadura hacia afuera.

Fuente: http://www.google.com/search 


\subsection{Placas de fundición:}

Elementos capaces de trasmitir las cargas axiales y los momentos flectores al suelo

Fig. 9. Placas de fundición.
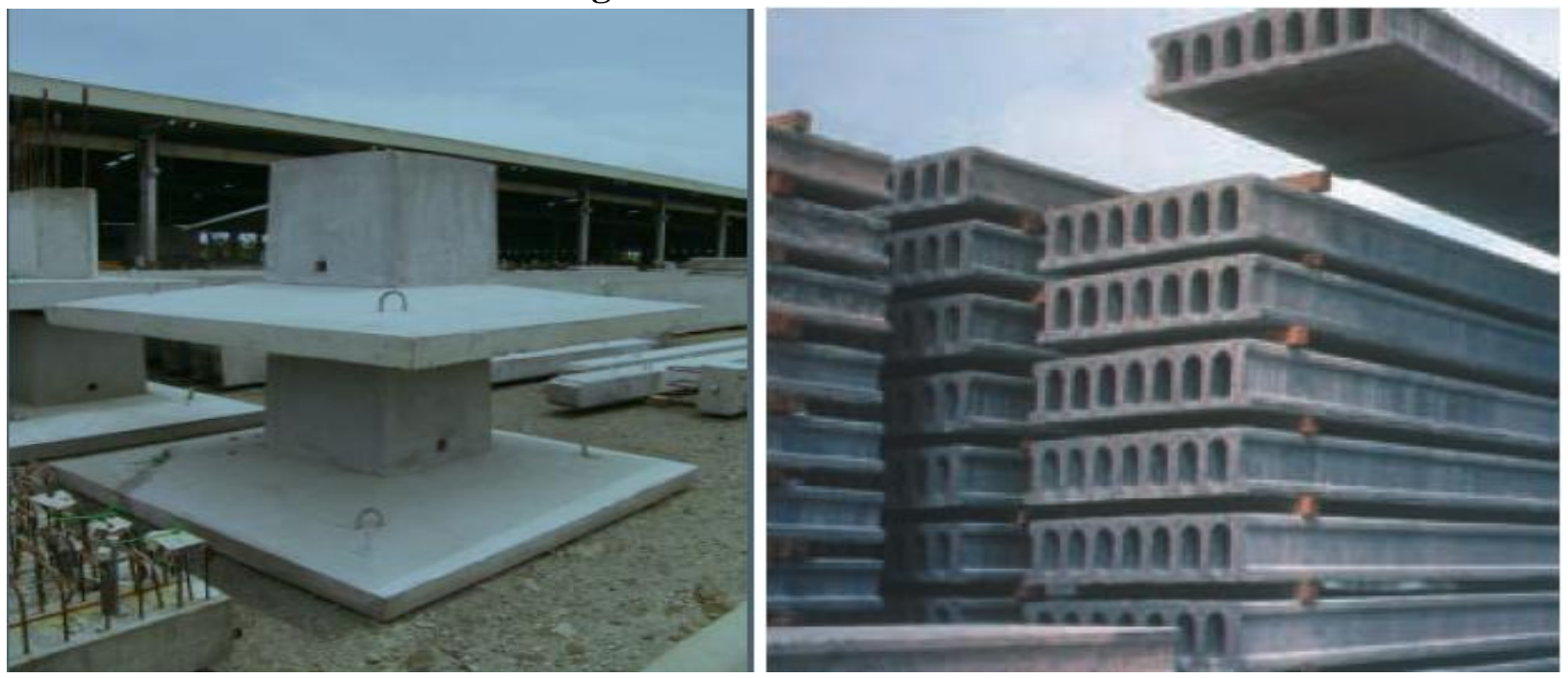

Fuente: http://www.google.com/search.

\subsection{Columnas:}

Pueden ser detalladas como elementos de ductilidad local moderada u óptima, la unión de la viga con el techo y con las vigas de amarre se detallan como conexión húmeda.

\subsection{Cubierta:}

La cubierta se realizara con el paneles nervados precisa, combinado con paneles traslucidos, estos paneles serán sujetos mediante pernos a las correas de la cubierta.

En los laterales y centro (encuentro de las cerchas) de la cubierta se colocan los canalones para el recogido de las aguas pluviales.

Fig 10. Tipo de cubiertas.

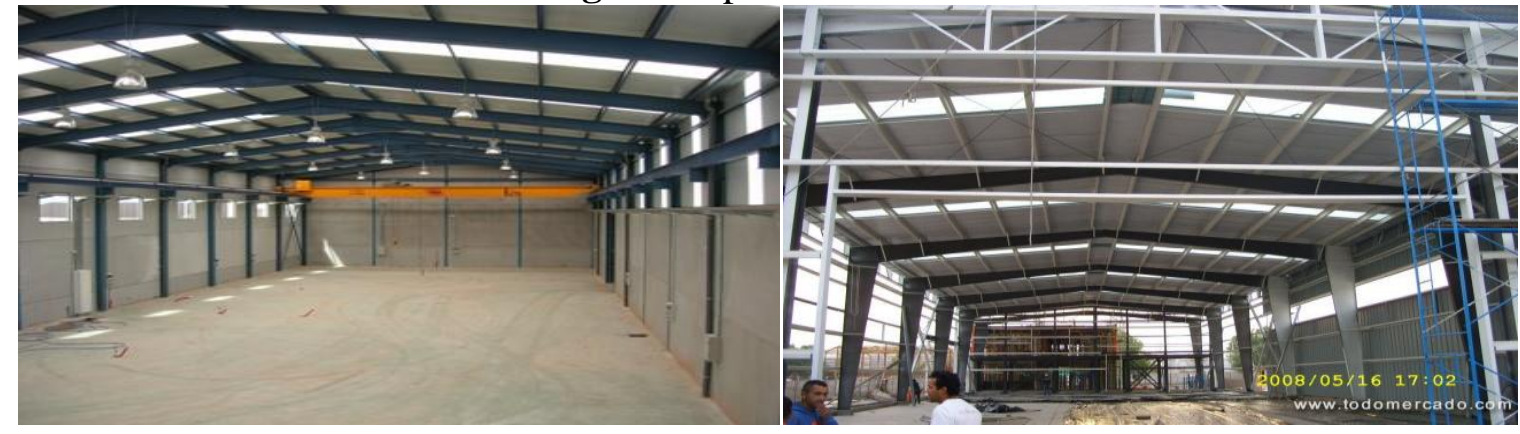

Fuente: http://www.google.com/search 


\subsubsection{Saneamiento:}

En los laterales y centro (encuentro de las cerchas) de la cubierta se colocan los canalones para el recogido de las aguas pluviales.

Se construirán sumideros de 0,5 x 0,9 y 9 x 0,5 metros, de profundidad variable dependiendo de la posición en los cambios de dirección. Se construirán con elementos prefabricados o realizados mediante obra cerámica, ladrillo, cubierta interiormente con cemento Portland.

\subsubsection{Pavimentos:}

El pavimento se realizará sobre el terreno con una base de grava de $15 \mathrm{~cm}$ para evitar la humedad. Una vez se coloca la grava se procede a colocar una malla de plástico denominada filtro anti textil. Este plástico evitará que las raíces sigan su camino. Una vez se haya colocado este plástico se procederá a poner una capa de $20 \mathrm{~cm}$ de hormigón HA-25-B-IIa vibrado y arremolinado al cuarzo $45 \mathrm{~kg} / \mathrm{m} 2$ con una malla electro soldada de 15 × 15 de diámetro 8.Esta adición previa al hormigón provoca que éste adquiera unas nuevas características más apropiadas para la utilización que se necesita, con una nueva dureza superior y mayor resistencia al desgaste.

\section{DISEÑO DE ALMACENES.}

Cada almacén es diferente, no vamos a encontrar un diseño ya hecho para el nuestro, si queremos rediseñarlo, mejorar en operatividad, o simplemente es un almacén nuevo hay una serie de principios y normas en las cuales tenemos que basarnos, siempre podemos ver algún almacén similar, pero al final tendremos que adaptarlo a nuestras necesidades, por lo cual siempre es conveniente tener en cuenta los principios que a continuación se exponen [40].

El conjunto hallado debe ahora traducirse en una representación detallada que muestre la forma y el tamaño exacto de cada departamento, el plan detallado de distribución, de los pasillos, el arreglo de las maquinas dentro de los centros de trabajo, el diseño de las aéreas de planta y de servicio personal [41].

\subsection{Almacenamiento en pilas.}

El almacenamiento en pilas o apilado es el que realiza mediante la colocación de las unidades de carga unas sobre otras directamente, es decir, sin más intermediación que el palé que les sirve de soporte. El acopio y almacenamiento, es necesario para tener el material y herramientas a nuestra disposición. Este se realizar de la forma más segura para evitar accidentes [42].

\subsection{Almacenamiento de estanterías}

Cuando la necesidad de almacenamiento en altura supera la capacidad de resistencia de apilado de las unidades de carga, o cuando se precisa cierta accesibilidad, mayor o menor en función de la necesidad de disponibilidad inmediata que se requiera del producto, es preciso recurrir al almacenamiento en estanterías [43].

Existen dos tipos diferentes de sistemas de almacenamiento en estanterías metálicas: almacenamiento móvil y almacenamiento estático [44]. 
El almacenamiento en estanterías y estructuras consiste en situar los distintos tipos y formas de carga en estantes y estructuras alveolares de altura variable, sirviéndose para ello de equipos de manutención manual o mecánica [45].

\subsection{Paletización compacta.}

El sistema de palatización compacta está desarrollado para almacenar productos homogéneos, con gran cantidad de paletas por referencia. Es el sistema que permite la máxima utilización del espacio disponible, tanto en superficie como en altura [46]. Esta instalación está constituida por un conjunto de estanterías, que forman calles interiores de carga, con carriles de apoyo para las paletas. Las carretillas penetran en dichas calles interiores con la carga elevada por encima del nivel en el que va a ser depositada [47].

\subsection{Carretillas elevadoras.}

Se denominan carretillas automotoras de manutención o elevadoras, todas las máquinas que se desplazan por el suelo, de tracción motorizada, destinadas fundamentalmente a transportar, empujar, tirar o levantar cargas. Para cumplir esta función es necesaria una adecuación entre el aparejo de trabajo de la carretilla (implemento) y el tipo de carga [48].

La carretilla elevadora es un aparato autónomo apto para llevar cargas en voladizo. Se asienta sobre dos ejes: motriz, el delantero y directriz, el trasero. Pueden ser eléctricas o con motor de combustión interna [49].

Estas carretillas son los elementos de manutención más utilizados en los almacenes convencionales por su buena relación entre precio y prestaciones, si bien también son las que peor aprovechan el espacio disponible. Para el apilado, una carretilla elevadora contrapesada utiliza un pasillo cuya dimensión se obtiene de sumar dos parámetros a su radio de giro [50].

Al circular las carretillas por el interior de las calles de almacenaje es necesario calcular los márgenes necesarios para que puedan trabajar con seguridad. Existen ciertas medidas que deben tenerse en cuenta a la hora de diseñar la instalación. La carretilla debe adaptarse a los locales en los que va a trabajar y a su vez el diseño de los ámbitos donde deba moverse la carretilla se ajustara a la característica de dichos ingenios.

a. Ancho total de la carretilla.

b. Estructura de protección de la operación.

c. Altura de la base de protección de la carretilla.

d. Altura de la base de protección de la carretilla.

e. Altura máxima de elevación.

El empuje de la carretilla se debe calcular de acuerdo con las normas y recomendaciones, un empuje de valor $35 \mathrm{~kg}$ producido por la carretilla elevadora y aplicado en el punto más favorable.

La flecha máxima de los carriles de apoyo de las paletas o deformación máxima se limita a la distancia entre apoyos / 200. Al tratarse de perfiles abiertos y de formas no simétricas, el cálculo de los carriles se efectúa mediante programas de elementos finitos. 
La estabilidad de las estanterías ha de garantizar su estabilidad tanto transversal como longitudinalmente. El plano transversal es el que incluye a los bastidores y el plano longitudinal es el perpendicular a las calles de almacenamiento.

Los bastidores formados por dos puntales con las diagonales, pies y accesorios correspondientes, van ranurados cada $50 \mathrm{~mm}$ para encajar en los largueros y soportes. El fondo del bastidor viene definido por las dimensiones de la calle de almacenaje, la altura, la medida y el peso de las paletas.

Las tolerancias en altura a considerar son:

$\mathbf{F}=$ altura nivel inferior y niveles intermedios $=$ altura paletas $+300 \mathrm{~mm}$.

$\mathbf{G}=$ altura nivel superior $=$ altura paletas $+200 \mathrm{~mm}$.

$\mathbf{H}=$ altura total = como mínimo la suma de todos los niveles.

Las cotas F, G y $\mathbf{H}$ han der ser múltiplos de $50 \mathrm{~mm}$. Para las tolerancias en fondo se ha de utilizar el mismo criterio que con carril GP-4.

El sistema de paletizacion compacta también permite construir almacenes auto portante, cuya principal característica es que no precisan la construcción de un edificio previo, con la consecuencia de reducción de tiempo y coste. En estas instalaciones, las estanterías soportan su propio peso, la carga de los productos que almacenan y las fuerzas adicionales correspondientes, como en un almacén clásico, así mismo, resisten el peso de la estructura y las fuerzas externas (viento, nieve, etc..) estos almacenes esta diseñados para trabajar tanto a temperatura ambiente como en frio, (cámaras frigoríficas).

El sistema de almacenaje compacto también es utilizado para funcionar con transelevadores, que desplazan sobre la plataforma de carga un carro satélite. Este, dirigido por el sistema informático que gestiona el almacén, se encargara de introducir y retirar las paletas de forma autónoma.

\section{CONCLUSIONES.}

En todo el mundo se reconoce hoy la amenaza que supone para nuestra civilización el cambio climático y la contaminación de la tierra, el agua y el aire. El desarrollo económico mal orientado consume recursos sin medida y afecta el medio ambiente, las consecuencias las vivirán las generaciones venideras y el coste para recuperar el estado de equilibrio del ecosistema puede ser mayor a largo plazo.

En la construcción y operación de los edificios se consume la mayoría de los recursos naturales y energéticos en la actualidad.

Es por ello que al diseñar Nuevas naves industriales debemos tener en cuenta herramientas que nos dé una guía de cómo realizar una construcción verdaderamente amigable con el medio ambiente y que a su vez sea sostenible, con respecto a factores de innovación de diseño, ahorro de agua potable, energía de eficiencia atmosférica, selección de materiales y calidad ambiental interior.

Con base en el presente escrito podemos evidenciar que hay normatividad amplia y específica sobre la forma de construir de manera correcta pero el hombre en su afán de generar beneficios monetarios no contempla esta normatividad y esto hace que el medio ambiente se afecte cada vez más. 


\section{BIBLIOGRAFIA.}

[1] Johnson y Sholes (2001) "Definición de Estrategia" 10p.

[2] Mintzberg H, Quinn J, (1995) "Estrategia" 5p.

[3] Antún, J.P. (1994) Logística: Una Visión Sistémica, Serie D-39 Instituto de Ingeniería, UNAM.

[4] Tompkins, J.A y White, J.A., (2003) "Planeación de Instalaciones”, New York. 15p.

[5] Martínez Robles, Agnessy Yerina, Tesis, "Control de Inventario con Analisis de la Demanda - Sistema Logistico" Capitulo II.

[6] Meyers, F.E., y Stephens, (2006) M.P., "Diseño de Instalaciones de Manufactura y Manejo de Materiales". $3^{\text {a }}$ edición. México D.F., Pearson educación de México.

[7] López López, Edgar Alejandro, (2009), Tesis, Maestría en Gerencia de Proyectos de Construcción, Cholula, Puebla, México a 5 de marzo de 2009.

[8] Bonilla García, Luis Enrique, (2006), Tesis, Licenciatura en Ingeniería Industrial con área de manufactura, Universidad de las Américas Puebla, Cholula, Puebla, México a 2 de mayo de 2006.

[9] Publicación: Revista Acción, “Zonas Francas”., Fecha de publicación: Marzo de 2008. Pagina consultada el 27 de marzo de 2011. En:

http://www.ccc.org.co/accion/094/f.html

[10] Publicación: Portafolio.com.co, “Zonas Francas: Una perspectiva más global”., Sección: Editorial - opinión. Fecha de publicación: 25 de octubre de 2009 Página consultada el 27 de marzo de 2011.

[11] Publicación: Diario La Republica, “Zonas Francas Permanentes”, Fecha de publicación: 11 de febrero de 2010. Página consultada el 27 de marzo de 2011.

[12] Licencias Urbanísticas. Manual para el Ciudadano. Alcaldía Mayor de Bogotá. Secretaría Distrital de Planeación. Colección Letras que Forman. Agosto 2006

[13] Publicación: Curaduría Urbana 3, Arq. Adriana López Moncayo., Página consultada el 26 de junio de 2011. En: http://www.curaduria3bogota.com/contenidos.php?Id_Categoria=146

[14] Agenda 21, (Junio de 2000) "Fondo Para el Medio Ambiente Mundial" 32p.

[15] Definición de Escombro, Wikipedia, Página consultada el 20 de julio de 2011. En: http://derechoencolombia.net/index.php?title=Escombro_-definici\%C3\%B3n_legal

[16] Alvira, Mary Isabel, (Mayo 17 de 2006), "La Responsabilidad Ambiental de las Empresas en Colombia" Universidad Nacional de Colombia, sede Manizales.

[17] Mejia, Elkin, (2003), "Protocolos Para El Manejo De Escombros y Materiales Sobrantes De Construcción” Universidad Nacional De Colombia, Sede Bogotá. 34p.

[18] DECRETO 357 DE 1997, (Mayo21), por el cual se regula el manejo, transporte y disposición final de escombros y materiales de construcción.

[19] Guía Ambiental de Construcción, Alcaldía Mayor de Bogotá, Secretaria Distrital de Ambiente, SDA, 2010

[20] Roca Ramón, Xavier, (2005), Tesis Doctoral, Estudio De La Aplicabilidad De Materiales Compuestos Avanzados En La Construcción De Edificios Industriales, Universidad Politécnica De Cataluña, Terrasa, España, Noviembre de 2005.

[21] Normatividad específica aplicable, Resolución 2413 de 1979, Decreto Distrital 959 de 2000, por el cual se reglamenta la publicidad Exterior Visual. Entre otras, Página consultada el 26 de junio de 2011, En:

http://www.alcaldiabogota.gov.co/sisjur/normas/Norma1.jsp?i=4718 19 
[22] Asociación Colombiana de Ingeniería Sísmica, (Septiembre de 2007), "Manual sobre Sismo Resistencia para Funcionarios de Planeación Municipal y otros profesionales de la ingeniería y arquitectura". Ministerio De Ambiente, Vivienda Y Desarrollo Territorial, 18p.

[23] Guevara Bayona, Gissele, Presentación Power Point, Ingeniera Industrial, Universidad Militar Nueva Granada, 2010.

[24] Arquitectura Solar Pasiva, Página consultada el 26 de junio de 2011, En:

http://es.wikipedia.org/wiki/Arquitectura_solar_pasiva

[25] Buenas Prácticas en la Construcción y en la Demolición, Construcción de edificios, Fundación Centro de Recursos Ambientales de Navarra, 2004.

[26] Certificación De Eficiencia Energética De Edificios, 2002, Información General.

[27] Dzul López, Luis Alonso, 2009, Tesis Doctoral, Los Costes De La Calidad En El Diseño De Proyectos De Construcción, Universitat Politecnica de Catalunya, Barcelona - España.

[28] Ezeldin, A. y Abu-Ghazala, H. (2007). Quality Management System for Design Consultants: Development and Application on Projects in the Middle East. Journal of management in engineering, 23(2), 75-87.

[29] Horcasitas M. E. (2001). La competitividad de la industria de la construcción. Cuadernos FICA, núm. 37, México.

[30] Orihuela A. Pablo, Orihuela A. Jorge, 2003, Constructabilidad En Pequeños Proyectos Inmobiliarios, VII Congreso Iberoamericano de Construcción y Desarrollo Inmobiliario - M.D.I. Perú 2003.

[31] McGeorge, D. \& Palmer, A. 1997, "Construction Management. New directions". Ed. Blackbell Scienck. Londres.

[32] USACH, "Lean Construction en Constructoras PYMES" Método Lean Construction.

[33] Silva Lira, Dorman Orlando, 2006, Consideraciones Sobre El Diseño Arquitectónico Con Estructuras De Acero, Universidad De San Carlos De Guatemala, Facultad De Arquitectura. Guatemala.

[34] Revista, Logismarket, Lula Gómez, 2009, Diseño De Naves Industriales, Barcelona España.

[35] Diccionario De Arquitectura Y Construcción, Pagina Consultada el 27 de junio de 2011, En: http://www.parro.com.ar/definicion-de-cubierta+en+diente+de+sierra

[36] Estructuras Metálicas, 2006, Ejecución de Nudos y Apoyos, Presentación Power Point, Naves Industriales, España.

[37] Piñar Pinedo, Paloma, 2010, Análisis De Viabilidad Económica y Optimización De Técnicas Energéticas y Constructivas Para Una Vivienda Aislada Autónoma. Universidad Pontificia Comillas, Ingeniera De Organización Industrial.

[38] Romo Proaño, Marcelo, Diseño De Naves Industriales Con Lamina Delgada En Acero, Escuela Politécnica Del Ejercito, Ecuador.

[39]. Diseño de un almacén, 2007, Zaragoza -España, www.gocisa.es

[40] Muños Cabanillas, Martin, Tesis, 2007, Diseño de Plantas, Portal del Sistema de Bibliotecas de la UNMSM, Peru.

[41] Guía de acopio de almacenaje, Pagina Consultada el 27 de junio de 2011, En:

http://www.grupovitruvio.org/guiasdidacticas/guiaacopios.pdf

[42] Logismarket, Mecalux (2011), "Soluciones de Almacenaje"., Estanterías para palatización compacta, Logismarket, Mecalux (2011), "Soluciones de Almacenaje”., pág. 11 - 32.

[43] Instituto Nacional De Seguridad E Higiene En El Trabajo, Guía Técnica señalización de Seguridad y Salud en el Trabajo (RD 485/1997)

[44]. Ministerio de trabajo y asuntos sociales, Almacenamiento en estanterías y estructuras, España. 
[45] Ministerio de trabajo y asuntos sociales, NTP 214, Carretillas elevadoras, España.

[46] Sistemas de almacenaje, Capítulo 7, Página consultada el 27 de junio de 2011.

[47] El Ministro de Obras Públicas, Transportes y Medio Ambiente, 1995, Estructuras De Acero En Edificación, Madrid - España.

[48] San Miguel Indurian, Jorge, Guía Para La Educación Y Evaluación De Riesgos En Carretillas Elevadoras, Universidad Pública De Navarra.

[49] Rey, María F. (Diciembre 15, 2008), "Encuesta Nacional Logística - Resultados del Benchmarking Logístico", Unidad Regional de Investigación del Center for Emerging Logistics and Supply Chains (CELSC).

[50] USAID, (09 de octubre de 2007) "La Competitividad Del Transporte Y Los Servicios Logísticos En El Comercio Exterior Colombiano" 